\title{
Impacto do grau de uveíte em diferentes tipos de úlceras de córneas em cães submetidos ao enxerto pediculado de conjuntiva bulbar - 34 casos
}

\author{
[Impact of uveitis score on different types of corneal ulcers in dogs subjected to the \\ bulbar conjunctival pedicle graft - 34 cases] \\ T.M. Amorim, A.P. Ribeiro, T. Ruiz, N.M.B. Dower, G. Madruga, D.C. Schroder, \\ G.B. Monteiro, B.E. Rodrigues
}

Universidade Federal de Mato Grosso - UFMT- Cuiabá, MT

\begin{abstract}
RESUMO
O presente trabalho objetivou avaliar os resultados de 34 olhos submetidos ao enxerto conjuntival pediculado (ECP) em ceratites ulcerativas profundas $(n=5)$, em ceratites ulcerativas com colagenólise $(n=2)$, descemetocele $(n=7)$, perfuração corneal $(n=15)$ e prolapso de íris $(n=5)$. Os impactos do grau de uveíte e da integridade da córnea foram correlacionados com presença e ausência de visão por tabelas de contingência. Raças braquicefálicas foram acometidas em $91,11 \%$ dos casos. O número de córneas consideradas perfuradas [20/34 (58,82\%)] foi maior que o de córneas íntegras [14/34 (41,17\%)]. Ao $50^{\circ}$ dia de pós-operatório, o número de olhos visuais que apresentavam córneas íntegras previamente às cirurgias $(\mathrm{n}=13)$ não diferiu significativamente dos olhos com córneas perfuradas $(\mathrm{n}=12)(\mathrm{P}=0,05)$. Avaliações relativas ao grau de uveíte, revelaram que a metade dos casos foi considerada severa [17/34 (50\%)] e na outra metade as uveítes foram consideradas discretas. Dos 17 casos que apresentaram uveíte severa, oito recuperaram a visão. Já nos 17 olhos onde a uveíte foi considerada leve, 15 mantiveram a visão ao final do período de avaliação. Apesar de a integridade da córnea não se correlacionar com a severidade da uveíte $(\mathrm{P}=0,48)$, constatou-se que o número de olhos visuais com uveíte discreta foi significativamente maior que os olhos com uveíte severa $(\mathrm{P}=0,006)$. Neste estudo, a taxa geral de sucesso visual após ECP foi de 73,52\% e a integridade da córnea não exerceu impacto significativo sobre a manutenção da visão. Todavia, olhos acometidos por uveítes severas apresentaram menor chance de recuperar a visão.
\end{abstract}

Palavras-chave: cão, iridociclite, microcirurgia, perfuração corneal

\begin{abstract}
The present study aimed to evaluate the results of 34 eyes of dogs presenting deep corneal ulcer $(n=5)$, colagenolytic corneal ulcer $(n=2)$, descemetocele $(n=7)$, corneal perforation $(n=15)$, and iris prolapse $(n=5)$ that were corrected by the bulbar conjuntival pedicle graft (CPG). The uveitis score and the corneal integrity were correlated with the presence or absence of vision by contingency tables. Brachycephalic breeds accounted for $91.11 \%$ of all cases. The number of perforated corneas [20/34 (58.82\%)] were higher than the non-perforated ones [14/34 (41.17\%)]. At post-operative day 50, the number of visual eyes with nonperforated corneas before surgery $(n=13)$ did not differ significantly from the eyes with perforated corneas $(n=12)(P=0.05)$. In half of the cases, uveitis score was considered severe [17/34 (50\%)], and in the other half, mild. Eight out of 17 eyes classified with severe uveitis regained vision. In 17 eyes where uveitis score was considered mild, 16 regained vision at the end of the study. Although corneal integrity was non-correlated with the uveitis score $(P=0.48)$, the number of visual eyes scored with mild uveitis were significantly larger than the eyes with severe uveitis $(P=0.006)$. In this study, the overall success visual rate after CPG was $73,52 \% \%$ and the corneal integrity did not play an important role regarding maintenance of vision. However, eyes presenting severe uveitis score had less chance to regain vision.
\end{abstract}

Keywords: dog, iridocyclitis, microsurgery, corneal perforation

Recebido em 16 de setembro de 2016

Aceito em 14 de novembro de 2016

E-mail: alexandre.aleribs@gmail.com 


\section{INTRODUÇÃO}

Afecções dos anexos oftálmicos, ceratoconjuntivite seca, infecções, traumatismos e anormalidades na aderência do epitélio corneal à membrana basal podem provocar ceratites ulcerativas. Nas ceratites superficiais, há perda somente do epitélio da córnea ou do estroma anterior, enquanto nas profundas, onde ocorre colagenólise secundária ao desequilíbrio entre metaloproteinases e seus inibidores teciduais, há envolvimento de mais da metade do estroma anterior. Nas ceratites superficiais e nas profundas, ocorre estímulo antidrômico do nervo trigêmeo, provocando quadros de uveíte anterior, com invasão de proteínas e células para o humor aquoso, com intensidade variável (Gelatt e Brooks, 2011).

Se não tratadas, as ceratites ulcerativas podem progredir até a membrana de Descemet e resultar em perfuração corneal. Nesse contexto, ocorre perda do humor aquoso, redução da câmara anterior e do diâmetro pupilar, podendo ocasionar sinéquia anterior, prolapso da íris e seclusão pupilar. Perfurações corneais não tratadas podem evoluir para endoftalmite, glaucoma ou Phthisis bulbi (Gelatt e Brooks, 2011).

Ceratites ulcerativas superficiais, geralmente, são tratadas com instilações de antibióticos, inibidores de metaloproteinases, midriáticos e anti-inflamatórios não esteroidais (Gelatt e Brooks, 2011). Nas profundas ou com perfuração da córnea, a despeito do tratamento médico, torna-se imperativa a correção cirúrgica. O enxerto de conjuntiva bulbar pediculado (ECP) foi introduzido em medicina veterinária há mais de 60 anos, sendo um procedimento factível para correção da ceratite ulcerativa profunda e puntiforme, mesmo na presença de perfuração corneal (Stern, 1950; Roberts, 1953). Na maior parte dos casos, o ECP é capaz de preservar a integridade do olho e da visão, além de permitir a visibilização da câmara anterior no acompanhamento pós-operatório (Gelatt e Brooks, 2011; Dorbandt et al., 2014). O ECP fornece aporte sanguíneo e celular, preenche defeitos tectônicos, oferece elementos do sistema imune e permite a entrega de antibióticos e outras substâncias administradas sistemicamente no leito ulcerado (Gelatt e Brooks, 2011; Dorbandt et al., 2014).
Além do ECP, membranas de matrizes acelulares de bexiga urinária ou da submucosa intestinal, bem como do pericárdio bovino, têm sido amplamente utilizadas na oftalmologia veterinária, com a vantagem de poderem ser adquiridas comercialmente (Dorbandt et al., 2014; Dulaurent et al., 2014; Balland et al., 2016; Chow e Westermeyer, 2016). Entretanto, reportou-se em cães que o uso dessas membranas pode não impedir a progressão da digestão do estroma corneal (Balland et al., 2016) e que o índice de deiscência é maior, quando comparado ao ECP (Chow e Westermeyer, 2016). Um estudo prévio reportou que córneas com ulcerações profundas e perfuradas, tratadas com ECP de forma isolada ou em associação com membranas acelulares, tiveram evolução clínica similar (Dorbandt et al., 2014). Embora extremamente empregado, nenhum estudo realizado no Brasil avaliou a eficácia do ECP na resolução de úlceras estromais puntiformes profundas e para o tratamento de perfurações corneais em cães. Tampouco nenhum estudo descrito na literatura avaliou o impacto do grau de uveíte induzida pela ceratite ulcerativa, relativamente à recuperação da visão. Dessa forma, o presente artigo visa apresentar resultados de 34 casos de ceratites ulcerativas de diversos tipos e de perfurações corneais (com e sem o prolapso da íris), em cães submetidas ao ECP. Ademais, objetivou-se avaliar possíveis correlações entre o impacto das uveítes e da integridade da córnea sobre a manutenção da visão.

\section{CASUÍSTICA}

A pesquisa foi aprovada pelo Comitê de Ética no Uso de Animais da Universidade Federal de Mato Grosso (UFMT), sob o protocolo de número 23108.163678/2016-75. Prontuários de cães, machos ou fêmeas, atendidos no setor de Oftalmologia Veterinária do Hospital Veterinário da Faculdade de Veterinária da UFMT , entre o período de janeiro de 2012 a maio de 2016, foram avaliados de forma retrospectiva. Todos os pacientes receberam um exame clínico e oftálmico completos. Foram incluídos no estudo aqueles que apresentaram ceratite ulcerativa puntiforne profunda (mais de $2 / 3$ do estroma) com (Fig. 1A) ou sem colagenólise (Fig. 1G) do estroma, exposição ou prolapso da membrana de Descemet e perfuração corneal, com (Fig. 1D) e sem prolapso da íris (Fig. 1J). 

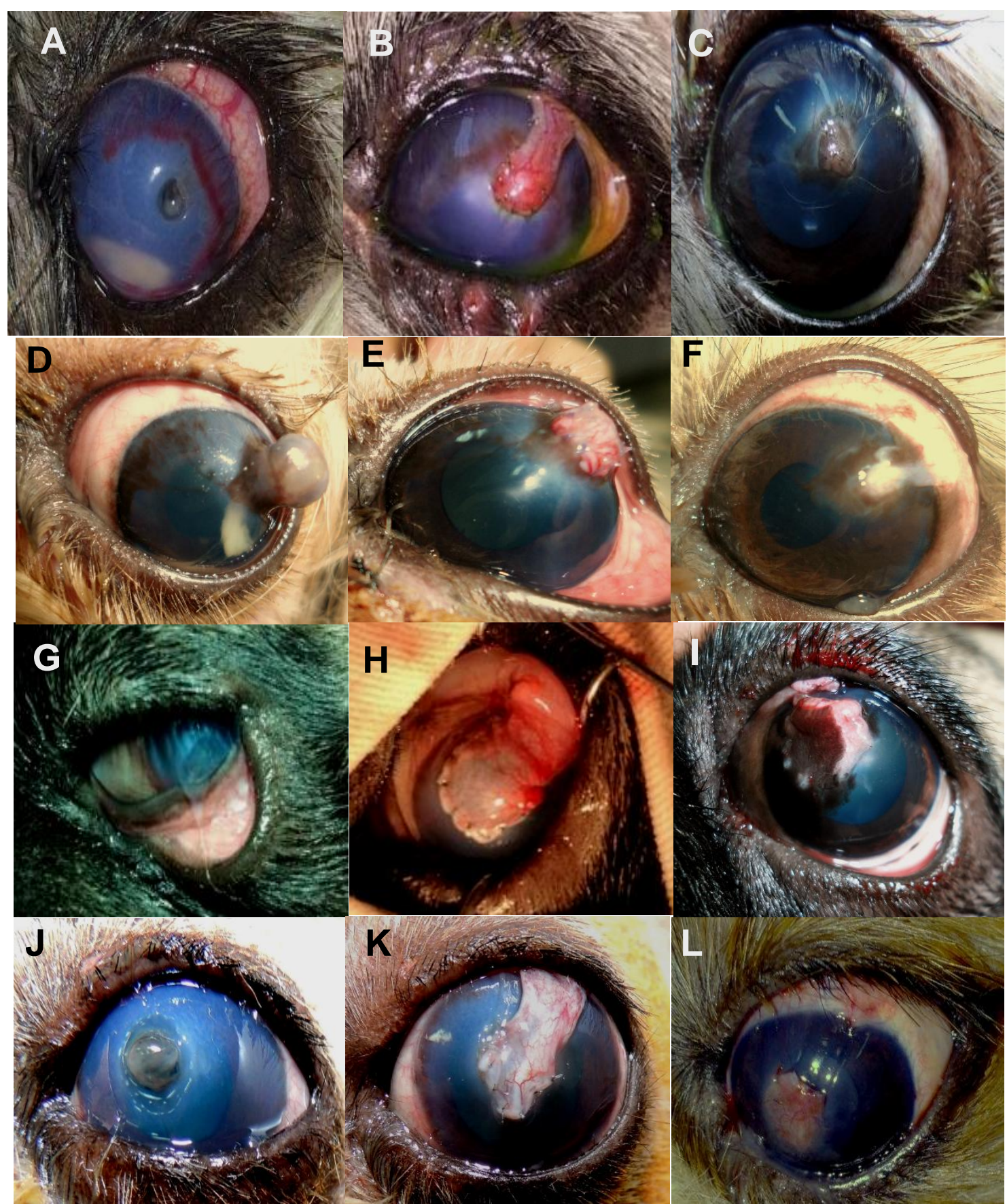

Figura 1. Imagens de cães com diferentes tipos de úlceras corneais submetidos ao enxerto pediculado de conjuntiva bulbar e após a sua retirada, decorridos 25 a 30 dias do procedimento A-L. Úlcera profunda e Figura 1. Imagens de cães com diferentes tipos de úlceras corneais submetidos ao enxerto pediculado de conjuntiva bulbar e após a sua retirada, decorridos 25 a 30 dias do procedimento A-L. Úlcera profunda e uveíte severa A-C (caso 7). Prolapso de íris crônico com uveíte severa C-D (caso 20). Úlcera colagenolítica e uveíte severa G-I (caso 23). Perfuração corneal tamponada com fibrina e uveíte discreta (caso 13) J-L.

Em todos os casos, previamente à cirurgia, as úlceras foram tratadas topicamente com antibióticos $\left(\right.$ Tobrex $^{\circledR}$, Alcon, São Paulo, Brasil), soro fetal bovino (Atená, Campinas, Brasil), 
atropina 1\% (Allergan, Guarulhos, Brasil) e antiinflamatório não esteroidal (AINE) (Acular ${ }^{\circledR}$, Allergan, Guarulhos, Brasil). No período pósoperatório, os pacientes mantiveram a terapia com antibióticos, atropina e AINE tópicos, por períodos variáveis, e AINE sistêmico (Maxicam $^{\circledR}$, Ouro fino, Cravinhos, Brasil), por via oral $(0,1 \mathrm{mg} / \mathrm{kg} / \mathrm{uma}$ vez ao dia/ quatro dias $)$.

Os proprietários relataram ter observado as lesões 24 a 48 horas antes da cirurgia, e em apenas um caso (20), a lesão já estava sendo tratada há 45 dias com ciprofloxacina e sulfato de condroitina. Os pacientes foram submetidos à anestesia geral inalatória, e os casos foram operados no mesmo dia, ou no dia seguinte ao atendimento. Em todos os casos, empregou-se a técnica do enxerto de conjuntiva bulbar pediculado (ECP) (Gelatt e Brooks, 2011). Os bordos das úlceras foram desbridados, o molde de fibrina foi desfeito em casos de perfuração e a câmara anterior foi lavada e preenchida com solução de ringer lactato acrescida de epinefrina $(0,05 \%)$. Em alguns casos, a íris encarcerada foi excisada com tesoura após divulsão com espátula de ciclodiálise. Posteriormente à confecção do ECP, a cápsula de Tenon foi liberada, o estroma conjuntival foi preso aos bordos da córnea com pontos simples separados com fio de náilon 9.0 ou de ácido poliglicólico 8.0, sob magnificação de 10-15 vezes, com auxílio de microscópio cirúrgico. Os enxertos conjuntivais foram removidos sob anestesia tópica ou geral, decorridos 25 a 30 dias da cirurgia. Foram considerados visuais, os pacientes que, após vedação do olho que não foi operado, apresentassem reflexo de ameaça positivo, seguissem o dedo do examinador e não colidissem com obstáculos ao teste de labirinto. Todos os casos operados apresentaram um mínimo de 50 dias de avaliação pós-operatória.

A despeito da profundidade da úlcera, o grau de uveíte também foi avaliado de forma qualiquantitativa, com biomicroscópio em lâmpada de fenda, segundo classificações descritas na literatura (Hendrix, 2013). Consideraram-se uveítes discretas aquelas em que o edema corneal, a presença de hipópio, de hifema e da turbidez do aquoso eram sutis ou moderados e em que se observava apenas tamponamento da perfuração com fibrina, bem como aquelas em que as estruturas intraoculares podiam ser visibilizadas (Fig. 1J). Consideraram-se uveítes severas aqueles casos em que o edema corneal e a turbidez do humor aquoso eram severos e em que se observavam fibrina, hipópio ou hifema de forma difusa, impedindo que as estruturas intraoculares pudessem ser visibilizadas (Fig. 1A).

Possíveis correlações entre a presença ou ausência de visão após as cirurgias com a integridade da córnea (perfuradas ou não) foram realizadas. A presença ou ausência de visão pósoperatória também foi correlacionada com o grau de uveíte atribuído antes das cirurgias (discreta ou severa), assim como a presença de pupila antes da cirurgia. Também se averiguaram possíveis correlações entre a integridade corneal e o grau de uveíte. O teste exato de Fisher foi utilizado para as avaliações em que em uma das células o número fosse menor que 5 , e o teste qui-quadrado se os valores de todas as células fossem iguais ou maiores que 5. A razão de chances, assim como o seu intervalo de confiança de $95 \%$, foi calculada pelo método de aproximação de Wolf. Em todas as análises, $\mathrm{P}<0,05$ foi considerado significativo.

\section{DISCUSSÃO E CONCLUSÕES}

$\mathrm{Na}$ série de 34 casos aqui apresentada, apenas um dos olhos apresentou-se doente; em 13 casos, o olho direito foi acometido (38,23\%), e em 21 casos o olho esquerdo (61,76\%) (Tab. 1). O número de cães braquicefálicos acometidos $(91,11 \%)$ foi maior que em outros dois estudos similares, que reportaram incidências de $43,83 \%$ e 68,42\% (Dorbandt et al., 2014; Chow e Westermeyer, 2016). Tais achados confirmam que a exoftalmia apresentada por essas raças favorece o acometimento da córnea por ceratites ulcerativas e não ulcerativas. $\mathrm{O}$ número de fêmeas $(52,94 \%)$ acometidas no presente estudo foi maior que o de machos $(47,05 \%)$, assim como a quantidade de Shi Tzus fêmeas $(n=16)$ em relação à de machos $(n=13)$ dessa mesma raça (Tab. 1). Tais resultados não corroboram os achados de outros dois estudos, os quais apontaram que machos da raça Shi Tzu apresentaram predisposição para o desenvolvimento de ceratite ulcerativa com perfuração corneal, comparativamente às fêmeas dessa mesma raça (Sanchez et al., 2007; Dorbandt et al., 2014). 
Impacto do grau...

Tabela 1. Resenha dos pacientes, tipo de úlcera, grau de uveíte e evolução de 33 olhos de cães operados pelo enxerto pediculado de conjuntiva bulbar.

\begin{tabular}{|c|c|c|c|c|c|c|c|}
\hline & Raça & Sexo & $\begin{array}{l}\text { Idade } \\
\text { (anos) }\end{array}$ & $\begin{array}{l}\text { Tipo de úlcera } \\
\text { (olho envolvido) }\end{array}$ & $\begin{array}{l}\text { Grau de } \\
\text { uveíte }\end{array}$ & $\begin{array}{l}\text { Pupila visível } \\
\text { antes da } \\
\text { cirurgia }\end{array}$ & $\begin{array}{c}\text { Visual ao } 50^{\circ} \\
\text { dia }\end{array}$ \\
\hline Caso 1 & Shih Tzu & Macho & 3,0 & Perfuração $(\mathrm{OE})$ & Leve & Sim & Sim \\
\hline Caso 2 & Shih Tzu & Fêmea & 8,0 & Úlcera colagenolítica $(\mathrm{OE})$ & Severa & Sim & Sim \\
\hline Caso 3 & Shih Tzu & Macho & 5,0 & Descemetocele (OE) & Leve & Sim & Sim \\
\hline Caso 4 & Shih Tzu & Macho & 6,0 & Úlcera profunda (OE) & Leve & Sim & Sim \\
\hline Caso 5 & Shih Tzu & Macho & 3,0 & Perfuração $(\mathrm{OE})$ & Severa & Sim & Sim \\
\hline Caso 6 & Shih Tzu & Fêmea & 1,0 & Perfuração (OD) & Leve & Sim & Sim \\
\hline Caso 7 & Shih Tzu & Fêmea & 2,0 & Úlcera profunda (OE) & Severa & Sim & Sim \\
\hline Caso 8 & Shih Tzu & Macho & 3,0 & Descemetocele (OE) & Severa & Sim & Sim \\
\hline Caso 9 & Shih Tzu & Fêmea & 4,5 & Perfuração (OD) & Leve & Não & Sim \\
\hline Caso 10 & Shih Tzu & Macho & 2,0 & Úlcera profunda (OE) & Leve & Sim & Sim \\
\hline Caso 11 & Shih Tzu & Fêmea & 1,5 & Perfuração (OE) & Severa & Não & Sim \\
\hline Caso 12 & Shih Tzu & Fêmea & 2,5 & Descemetocele (OE) & Leve & Sim & Sim \\
\hline Caso 13 & Shih Tzu & Fêmea & 3,0 & Perfuração (OE) & Leve & Sim & Sim \\
\hline Caso 14 & Shih Tzu & Macho & 6,0 & Perfuração (OD) & Severa & Não & Não \\
\hline Caso 15 & Shih Tzu & Fêmea & 3,0 & Perfuração (OD) & Severa & Não & Não \\
\hline Caso 16 & Shih Tzu & Fêmea & 2,0 & Perfuração (OE) & Severa & Não & Não \\
\hline Caso 17 & Shih Tzu & Fêmea & 3,2 & Perfuração (OD) & Severa & Sim & Não \\
\hline Caso 18 & Shih Tzu & Fêmea & 4,0 & Descemetocele (OE) & Leve & Sim & Sim \\
\hline Caso 19 & Shih Tzu & Fêmea & 2,5 & Perfuração (OD) & Leve & Sim & Sim \\
\hline Caso 20 & SRD & Macho & 7,8 & Descemetocele (OE) & Leve & Sim & Sim \\
\hline Caso 21 & SRD & Fêmea & 4,0 & Prolapso da íris (OD) & Severa & Sim & Não \\
\hline Caso 22 & Pug & Fêmea & 6,0 & Descemetocele (OE) & Severa & Sim & Não \\
\hline Caso 23 & Pug & Macho & 2,0 & Úlcera colagenolítica $(\mathrm{OE})$ & Severa & Não & Sim \\
\hline Caso 24 & PiBull & Fêmea & 4,0 & Descemetocele (OD) & Severa & Sim & Não \\
\hline Caso 25 & SRD & Macho & 1,0 & Perfuração (OD) & Severa & Não & Sim \\
\hline Caso 26 & Shi Tzu & Fêmea & 4,0 & Perfuração (OE) & Leve & Sim & Sim \\
\hline Caso 27 & Shi Tzu & Macho & 5,0 & Prolapso de íris (OD) & Severa & Não & Não \\
\hline Caso 28 & Shi Tzu & Macho & 1,5 & Prolapso da íris (OD) & Severa & Não & Sim \\
\hline Caso 29 & Shi Tzu & Macho & 2,0 & Perfuração (OE) & Leve & Sim & Não \\
\hline Caso 30 & Shi Tzu & Macho & 0,83 & Úlcera profunda (OE) & Leve & Sim & Sim \\
\hline Caso 31 & Shi Tzu & Macho & 0,66 & Úlcera profunda (OE) & Leve & Sim & Sim \\
\hline Caso 32 & Shi Tzu & Fêmea & 1,0 & Perfuração (OD) & Severa & Não & Não \\
\hline Caso 33 & Shi Tzu & Macho & 2,70 & Prolapso de íris (OD) & Leve & Sim & Sim \\
\hline Caso 34 & Shi Tzu & Fêmea & 4,00 & Prolapso de íris (OE) & Leve & Sim & Sim \\
\hline
\end{tabular}

$\mathrm{O}$ presente trabalho demonstrou que o ECP suturado diretamente ao leito ulcerado ou perfurado da córnea apresentou eficácia geral de $73,52 \%$ na recuperação do tecido corneal doente e na manutenção da visão (Tab. 1). Outro estudo, em que a mesma técnica foi empregada em 37 olhos acometidos por afecções corneais similares àquelas aqui demonstradas, apresentou taxa geral de sucesso visual de 97\% (Dorbandt et al., 2014). Embora não reportadas no estudo de Dorbandt et al. (2014), tais discrepâncias em relação aos resultados aqui apresentados podem ser atribuídas ao tempo decorrido entre a ocorrência da lesão e a correção cirúrgica.

Membranas constituídas de matriz acelular de bexiga urinária ou de submucosa de intestino têm sido amplamente utilizadas na oftalmologia veterinária, com a vantagem de poderem ser adquiridas comercialmente (Dorbandt et al., 2014; Balland et al., 2016; Chow e Westermeyer,
2016). Dorbandt et al. (2014) reportaram que o sucesso cirúrgico e visual não diferiu em cães tratados com ECP de forma isolada ou associado ao enxerto de membrana de submucosa. Todavia, nenhum estudo em que membranas acelulares foram utilizadas comparou seus resultados, separadamente, com os resultados do sucesso cirúrgico em cães operados pelo ECP (Dorbandt et al., 2014; Balland et al., 2016; Chow e Westermeyer, 2016). Reportou-se, em cães, que o uso de membranas acelulares pode não impedir a progressão da digestão do estroma corneal (Balland et al., 2016) e que o índice de deiscência é maior, quando comparado a outras técnicas convencionais que empregam conjuntiva (Chow e Westermeyer, 2016). No presente estudo, apenas dois casos apresentaram colagenólise do estroma corneal antes da cirurgia. Embora ambos recuperassem a visão ao final do estudo, em apenas um observou-se deiscência do ECP. Admite-se que a falha do 
ECP em um dos casos de colagenólise possa ser atribuída ao não cumprimento de normas relativas aos cuidados pós-operatórios pelo proprietário.

Relativamente aos pacientes acometidos por descemetocele, os percentis de recuperação visual foram inferiores $(71,42 \%$ vs. $100 \%)$ aos reportados por outros autores (Dorbandt et al., 2014). Admite-se, entretanto, que os dois cães (casos 22 e 24) dessa população (descemetocele) que não recuperaram a visão ao final do estudo apresentavam-se visuais na primeira semana de pós-operatório. Dessa forma, não se apontou o insucesso cirúrgico do ECP como causa da perda de visão, e sim a descontinuidade do tratamento prévio de ceratoconjuntivite seca, que evoluiu para ceratite pigmentar. Todavia, neste estudo e em outro, admite-se que o ECP parece ter sido mais eficaz que o enxerto de membrana acelular para correções de olhos acometidos por descemetoceles (Dorbandt et al., 2014). Já nos cinco pacientes acometidos por ceratite ulcerativa profunda, sem exposição da membrana de Descemet, o ECP foi $100 \%$ eficaz na recuperação da visão, à similitude do reportado na literatura (Scagliotti, 1988; Dorbandt et al., 2014).

Em um estudo que avaliou diferentes tipos de ceratites ulcerativas em cães reportou-se que, do total de olhos perfurados observados, apenas $40 \%$ tornaram-se visuais após a cirurgia de ECP (Scagliotti, 1988). Outro estudo mais recente mostrou não haver impacto significativo entre integridade corneal e o sucesso visual de cães submetidos ao ECP (Dorbandt et al., 2014). Todavia, Dorbandt et al. (2014) relataram que 4/5 dos casos malsucedidos apresentavam perfuração corneal. No presente estudo, o número de olhos visuais ao $50^{\circ}$ dia de pósoperatório, que apresentavam córneas íntegras previamente às cirurgias $(n=13 / 14)$ não diferiu significativamente dos olhos com córneas perfuradas $(\mathrm{n}=12 / 20)(\mathrm{P}=0,05$; razão de chances $(\mathrm{RC})=0,11$; intervalo de confiança (IC) de $95 \%=0,01$ a 1,06$)$. Das 20 córneas perfuradas, cinco apresentavam prolapso da íris e em três observou-se recuperação da visão, assemelhando estes resultados com os previamente reportados (Scagliotti, 1988; Dorbandt et al., 2014). Considerando o número total de córneas perfuradas tamponadas por fibrina ou com prolapso da íris, o sucesso visual no presente estudo foi de $60 \%$ vs. $92 \%$ observado em estudo prévio (Dorbandt et al., 2014).

Estudos prévios em cães com ceratite ulcerativa e perfuração corneal não indicaram correlações entre o grau de uveíte e o sucesso visual após enxertia de membrana acelular, pericárdio bovino ou ECP (Scagliotti, 1988; Dorbandt et al., 2014; Dulaurent et al., 2014; Balland et al., 2016; Chow e Westermeyer, 2016). Na série de casos aqui relatada, dos 17 olhos que apresentaram uveíte severa, apenas oito recuperaram a visão, a despeito do tratamento tópico e sistêmico com AINEs. Constatou-se que o número de olhos que apresentavam uveíte discreta e recuperaram a visão ao final do período de avaliação foi significativamente maior que daqueles que apresentavam uveíte severa $(\mathrm{P}=0,006 ; \mathrm{RC}=0,05$; $\mathrm{IC}$ de $95 \%=0,005$ a 0,51). Todavia, não se observou correlação entre integridade corneal e severidade da uveíte $(\mathrm{P}=0,48 ; \mathrm{RC}=0,61 ; \mathrm{IC}$ de $95 \%=0,15$ a 2,43$)$. Dos nove olhos que permaneceram cegos, dois foram enucleados devido à evolução para glaucoma (casos 18 e 21) e outro devido a sinais de desconforto associados ao Phthisis bulbi (caso 27). Admite-se que, quando não controladas, uveítes podem evoluir para as complicações descritas no presente estudo (Hendrix, 2013). Outro estudo realizado em cães não descreveu tais complicações após enxertia de membrana acelular e ECP, porém não se comentou sobre o grau de uveíte (Dorbandt et al., 2014). Todavia, evolução para glaucoma ou Phthisis bulbi foi relatada em cinco olhos de cães que apresentavam perfuração ou úlcera colagenolítica (tratados com membrana acelular), em que o escore de uveíte geralmente é elevado (Chow e Westermeyer, 2016)

Embora na presente pesquisa não tenha sido observada correlação entre visibilidade précirúrgica (devido à presença de pupila) e recuperação visual pós-cirúrgica $(\mathrm{P}=0,08$; $\mathrm{RC}=3,80$; IC de $95 \%=0,77$ a 18,52), dos 17 olhos que apresentavam uveíte severa, 11 apresentaram pupila não responsiva à terapia midriática tópica ou após a injeção intracameral de solução de epinefrina $0,05 \%$. Mesmo sem ausência de correlação significativa, os autores admitem que o encarceramento da íris na perfuração ou a formação de sinéquias anteriores são fatores negativos para a manutenção da visão (Hendrix, 2013). No estudo de Dorbandt et al. 
(2014), os autores descrevem que pupilas encarceradas nas perfurações corneais foram descoladas pelo auxílio de viscoelástico intracameral. Embora a literatura veterinária recomende essa conduta, no presente estudo, não se adotou essa técnica (Gelatt e Brooks, 2011). Nos casos de encarceramento de íris, foi realizada apenas iridectomia, combinada com a injeção de solução de epinefrina $0,05 \%$ para preenchimento da câmara anterior, sendo efetiva em $81,23 \%$ dos casos.

Clinicamente, o grau de uveíte ativa pode ser classificado como discreto, moderado ou severo, devido à presença ou ausência de sinéquia, do grau de turbidez do humor aquoso, do edema de córnea e da injeção ciliar (Hendrix, 2013). Na série de casos aqui reportada, as uveítes moderadas foram classificadas como discretas e optou-se por classificar as uveítes em apenas dois subgrupos (discreta ou severa) para não comprometer as avaliações estatísticas. Todavia, os autores acreditam que o agrupamento não tenha comprometido as avaliações, haja vista que apenas 1/17 dos olhos classificados como uveíte discreta não recuperou a visão no pós-operatório. Mesmo assim, admite-se que erro estatístico do tipo II possa ter ocorrido em algumas avaliações, devido ao número reduzido de pacientes.

Conclui-se que, na série de 34 casos aqui relatados, o ECP foi factível em recuperar a visão em $100 \%$ dos olhos acometidos por úlceras profundas e colagenolíticas, em $71,42 \%$ dos olhos acometidos por decementocele e em $60 \%$ dos olhos acometidos por perfurações e prolapso da íris. Embora a integridade da córnea não tenha exercido impacto significativo sobre a manutenção da visão, os olhos acometidos por uveítes severas apresentaram significativamente menor chance de recuperar a visão.

\section{REFERÊNCIAS}

BALLAND, O.; POINSARD, A.S.; FAMOSE, F. et.al. Use of a porcine urinary bladder acellular matrix for corneal reconstruction in dogs and cats. Vet. Ophthalmol., v.19, p.454-463, 2016.
CHOW, D.W.Y.; WESTERMEYER, H.D. Retrospective evaluation of corneal reconstruction using ACell $\mathrm{Vet}^{\mathrm{TM}}$ alone in dogs and cats: 82 cases. Vet. Ophthalmol., v.19, p.357366, 2016.

DORBANDT D.M.; MOORE, P.A.; MYRNA, K.E. Outcome of conjunctival flap repair for corneal defects with and without an acellular submucosa implant in 73 canine eyes. Vet. Ophthalmol., v.18, p.1-7, 2014.

DULAURENT, T.; AZOULAY., T.; GOULLE, F. et al. Use of bovine pericardium (Tutopatch ${ }^{\circledR}$ ) graft for surgical repair of deep melting corneal ulcers in dogs and corneal sequestra in cats. Vet. Ophthalmol., v.17, p.91-99, 2014.

GELATT, K.N.; BROOKS, D. Surgery of the cornea and sclera. In: GELATT, K.N.; GELATT, J.P. (Eds.). Veterinary ophthalmic surgery. 3.ed. Oxford: Saunders Elsevier, 2011. p.191-236.

HENDRIX, D.V.H. Diseases and surgery of the canine anterior uvea. In: GEATT, K.N. (Ed.). Veterinary ophthalmology, 5.ed. Iowa: Blackwell-Wiley, 2013. p.1146-1198.

ROBERTS, S.R. The conjunctival flap operation in small animals. J. Am. Vet. Med. Assoc., v.122, p.86-90, 1953.

SANCHEZ, R.F.; INNOCENT, G.; MOULD, J. et al. Canine keratoconjunctivitis sicca: disease trends in a review of 229 cases. J. Small Anim. Pract., v.48, p.211-217, 2007.

SCAGLIOTTI, R.H. Tarsoconjunctival island graft for the treatment of deep corneal ulcers, desmetocoeles, and perforations in 35 dogs and 6 cats. Semin. Vet. Med. Surg. Small Anim., v.3, p.69-76, 1988 .

STERN, A.I. Conjunctival flap operation. J. Am. Vet. Med. Assoc., v.116, p.44-45, 1950. 